\title{
Meristematic Tissue in Pinus Koraiensis
}

\author{
Zhu Hua Ning, Xing Yuan He, and Kamran Abdollahi
}

Chinese Academy of Sciences, Institute for Applied Ecology, Shen Yang, China

Southern University, Urban Forestry Program, Baton Rouge, LA 70813

In Northeast of China, Pinus koraiensis is a widely used urban tree species. It is a cold hardy evergreen tree with magnificent form. To meet the high demand on this tree for urban planting, this tree has been massively propagated through seeding in nurseries. To obtain information in seed germination and seedling survival, a series of studies has been done. This paper reports the activities, characteristics, and function of the root apex meristematic tissue of germinated seeds.

Tissue samples were collected from the root apex two days after seed germination. They were fixed over night at $4 \mathrm{C}^{0}$ in $4 \%$ glutaraldehyde buffered with a phosphate buffer at a $\mathrm{pH}$ of 7.2. Then samples were dehydrated by using a graded enthanol series and embedded in Spurr's epoxy resin. Sections were cut and positive stained with uranyl acetate followed by lead citrate. Both Hitachi H5-9 transmission electron microscope and AEI EM7 1.2 MV high voltage electron microscope were used for examination.

The most significant function of the root apex meristem was that it carried on cell division and differentiation activities. Two days after seed germination, most mitotic activities concentrated in the root apical meristem region (Fig 1). At this stage, tissue from hypocotyls of the root apex revealed a remarkable pattern of vascular differentiation (Fig. 2). The phloic and xylic parts of the procambium of the hypocotyl had been morphologically differentiated into protophloem and protoxylem, respectively.

As the seed germinated, the processes of cell elongation and wall formation became of central importance. The vesicles derived from the Golgi apparatus played an important role in the formation of the middle lamella and plasma membrane on either side of the cell plate (Fig. 3). Following the formation of the middle lamella, each protoplast of two daughter cells deposited a primary cell wall next to the middle lamella. The plasmodesmata were also formed during cell wall formation.

During seed germination, a great amount of protein that accumulated in the tissues was broken down and utilized as energy. After protein has been dissolved in the protein bodies, vacuoles were formed (Fig.4). The vacuoles had irregular, even convoluted tonoplasts. The large vacuoles were formed by the fusion together of smaller ones. As germination proceeded, the tissue gained in overall starch content (Fig. 4). Starch was usually stored as starch grains. The most conspicuous of the cytoplasmic components of the cells were the starch grains. The grains were ovoid when large, and nearly spherical when small.

Other cell types found in the root apex were parenchyma cells. They were large, relatively undifferentiated, and consisted of a thin wall, a thin layer of parietal cytoplasm, a nucleus, and a large central vacuole with watery contents. 


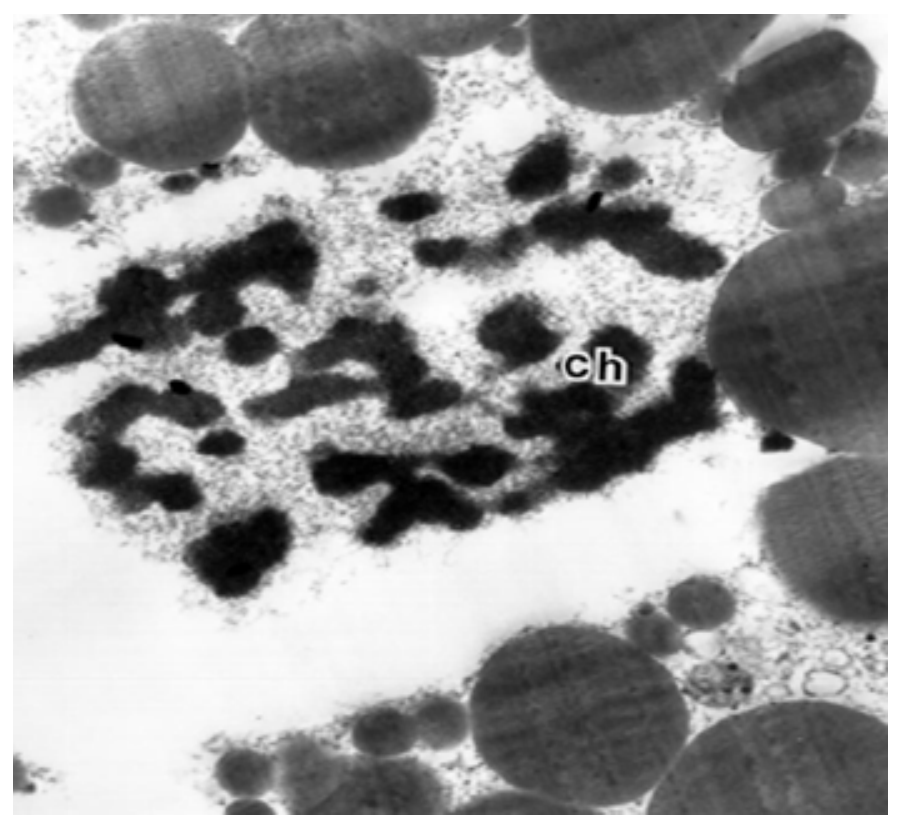

Figure 1. A cell of root apical meristem two days after seed germination. Note the cell is in the late prophase to early metaphase of mitosis. The chromosomes (ch) have condensed and the nucleolus and the nuclear envelope have disappeared but the spindle fibers are as yet barely visible. X 3000.

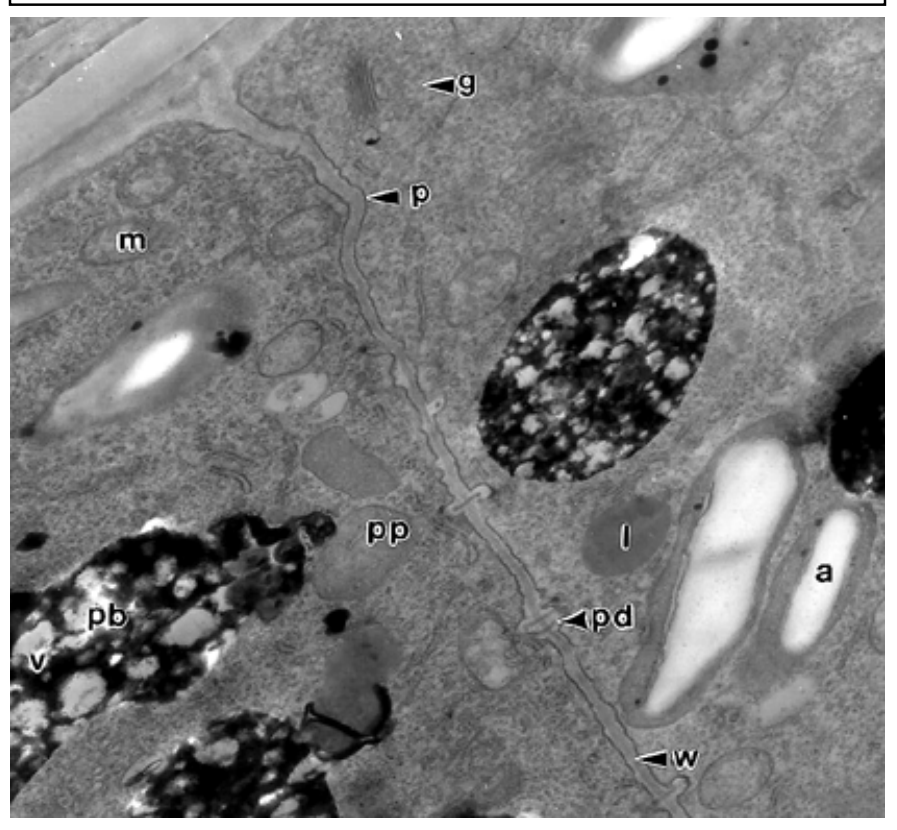

Figure 3. A section of root meristematic tissue two days after seed germination. g, Golgi body; $p$, plasma membrane; w, primary cell wall; pd, plasmodesmata; $\mathrm{pb}$, protein body; v, vacuole; a, amyloplasts; 1 , lipid droplets; m, mitochondria; pp, proplastids. X 5000.

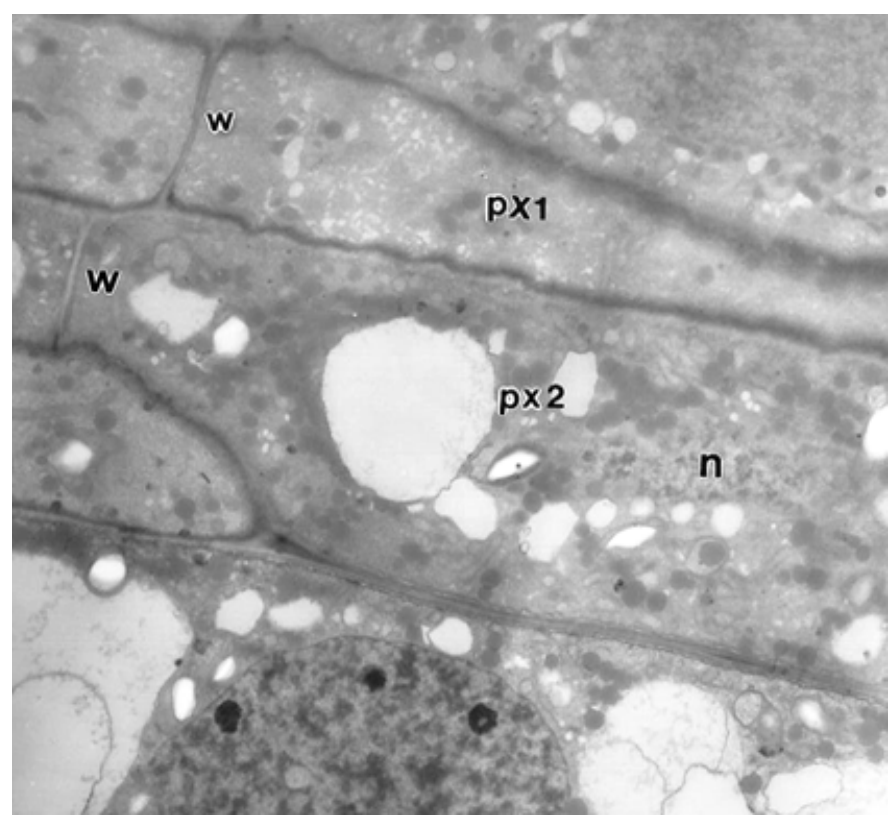

Figure 2. A longitudinal section of hypocotyls of the root apex. Protoxylem cells (px1 and px2) are recognized. The cell walls of protoxylem cell 1 (px1) have been pulled by elongation of the surrounding cells. Protoxylem cell 2 (px2) developed later than the protoxylem cell 1 . The end wall $(\mathrm{w})$ of protoxylem cell separates it from the next cell. Nucleus (n) and cytoplasm are still present in the protoxylem cell 2. X1600.

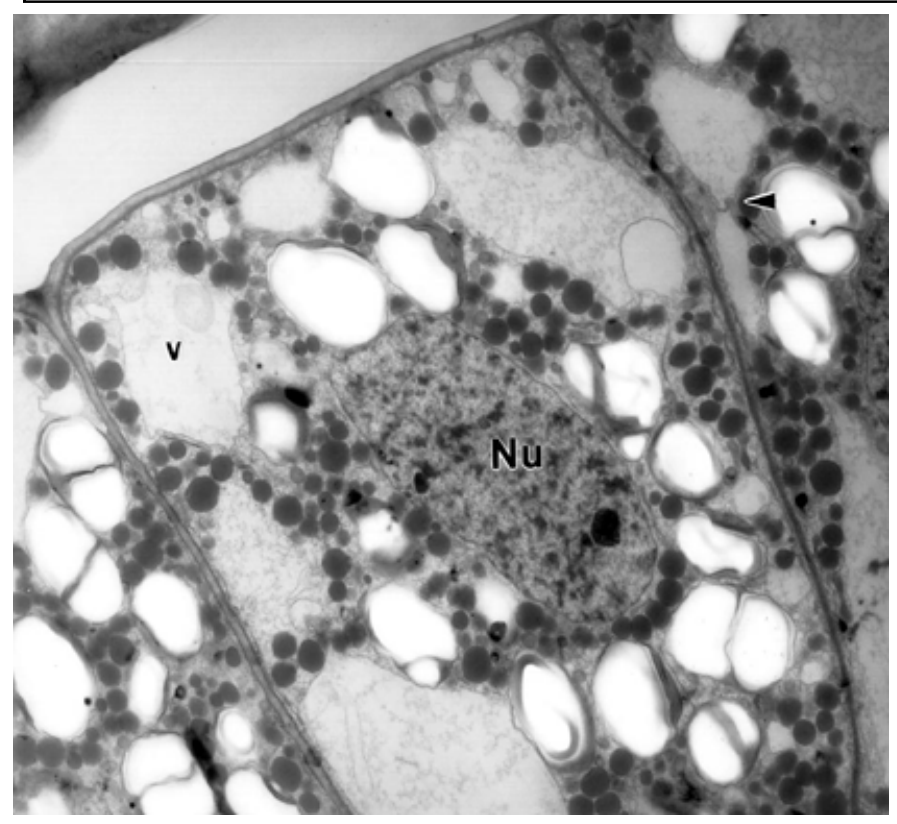

Figure 4. A section sliced through the mid-region of the cells in the hypocotyls of the root apex. Cells are thin-walled and have large nuclei $(\mathrm{Nu})$. The vacuoles (v) have irregular, even convoluted tonoplasts. Note two vacuoles on the upper right (arrow) are fusing together to form a larger vacuole. X 1600 